\title{
An Information Hiding Framework for Lightweight Mobile Devices with Digital Camera
}

\author{
Subhamoy Maitra ${ }^{1}$, Tanmoy Kanti Das ${ }^{2}$, and Jianying Zhou ${ }^{2}$ \\ 1 Applied Statistics Unit, Indian Statistical Institute, \\ 203, B T Road, Calcutta 700 108, India \\ subho@isical.ac.in \\ 2 Infocomm Security Department, Institute for Infocomm Research, \\ 21 Heng Mui Keng Terrace, Singapore 119613 \\ \{tkdas, jyzhou\}@i2r.a-star.edu.sg
}

\begin{abstract}
In this paper we present a paradigm for robust information hiding strategy designed with proper cryptographic and error correcting frameworks in mind that works for low end hardware devices like mobile phones with camera.
\end{abstract}

Keywords: Steganography, Digital Watermarking, Information Security, JPEG Compression, Mobile Devices.

\section{Introduction}

Information hiding has recently gained a lot of attention. The two main directions of information hiding are Steganography and Digital Watermarking. Though in terms of application these are completely different, the basic technical idea is same: to hide secret information in an innocent looking cover media, and the most frequently used cover media is image (in some cases audio also). In this paper we will consider image as the cover media.

In Steganography, secret message (binary string) is inserted in an image in a manner such that the visual quality of the image does not change at all. From the attacker side, the problem is given an image and the message embedding algorithm whether one can understand if there is any secret message contained in the cover media. Recovering the secret message is clearly a stronger attack, but this is elusive as the designer will not embed the plain message text in the cover, the encrypted version of the plain text will be used to communicate the secret. The state of the art encryption mechanisms are extremely robust and it is believed to be impossible to decrypt the encrypted message without knowing the secret key. Thus design of a robust steganographic scheme should satisfy that attacker will not be able to decide whether a media contains some secret information.

Digital watermarking is used for copyright protection. Given an image, the owner inserts a secret signature in it without making any visual change (we are only considering invisible watermarks). This is called the watermarked copy of the original image. If the watermarked copy is available from any resource, then 
the owner should be able to extract the secret signature to prove the copyright. Further, the owner may put buyer specific signatures in different watermarked copies of the same image. In that case the owner can prove the copyright as well as one can also pin down the buyer also, which is called fingerprinting. The attack model considers that the algorithms for watermark embedding and extraction are known and the attacker likes to destroy the watermark signal from the image. The robustness of the watermarking strategy is primarily evaluated on the following two criteria: (i) how well the malicious buyer is identified (who has intentionally attacked the watermarked image) and (ii) how infrequently an honest buyer is wrongly implicated. We like to refer [1/2/315] and references in these materials for more details on steganography and digital watermarking and their cryptanalysis.

In the last few years there is an enormous growth in mobile communication and mobile equipments. Mobile phones of different brands are available in the market which carry digital camera of considerably good quality. There are now standards proposed by Third-Generation Partnership Project (3GPP) and Internet Engineering Task Force (IETF) for voice calls enriched with live or pre-recorded video clips (see http://www.nokia.com). Given this growth there is a natural need to hide information in the images generated or stored in mobile devices, from both the angles of steganography and digital watermarking. Naturally JPEG CODEC is available in most of the processors, as example S1D13710/712 (see EPSON ED Journal, Vol 30, 2002). Thus we need a robust embedding scheme that uses JPEG image as the cover media. That is why we resort to the recent embedding scheme presented in 2 .

Now we present the information hiding framework. In this case we will first take a message string $M$ and encrypt it using a key $K$ to get the cipher string $C$. Then $C$ is encoded to generate an expanded string $C_{E}$. This is done for better error correction. Then the expanded string $C_{E}$ is embedded to a cover image $I$ using an image key $K_{I}$ to generate a visually indistinguishable image $I^{\prime} . I^{\prime}$ is then communicated through an open channel. At the receiver side $I^{\prime \prime}$ (a modified version of $I^{\prime}$ due to transmission error, signal processing, intentional attack etc.) is received and an extraction method is used (with the image key $K_{I}$ ) to get the encoded string $C_{E}^{\prime}$ (may not be same as $C_{E}$ ). Then we use the decoding strategy to get $C^{\prime}$ (may not be same as $C$ ) and then decryption is done using the key $K$ to get the message $M^{\prime}$ (may not be same as $M$ ).

Towards the design, we consider that an adversary will know the complete algorithm with all the parameters except the keys $K, K_{I}$. For steganographic purposes we need: (i) nobody can identify whether a message is embedded in $I^{\prime}$, (ii) the recovered message $M^{\prime}$ should be as close as possible to $M$. For watermarking purposes a good correlation between $M^{\prime}, M$ is sufficient for the owner to prove the copyright and identify the malacious buyer.

One may refer to [5] for a similar outline of a steganographic scheme in general. However, our attempt is more concrete in terms of design and implementation. We like to highlight the following issues that are addressed in this paper. We also like to mention that there are number of steganographic schemes 
that use LSB insertion, masking and filtering etc. However, since the schemes are basically based on image processing techniques only, they are not as robust as the scheme we propose here.

- There is an enormous development in mobile equipments where images can be captured and communicated. The hardware and software facilities are constrained in this environment. We like to explain a scheme which can be implemented in lightweight devices with sufficient amount of security for the purpose of information hiding.

- Note that even if a cryptosystem is designed very carefully, the security of the system is a conjecture. That is one needs to specify the scheme and then it is open to the community for cryptanalysis. Here we present a specific scheme with all the parameters so that the community may pay attention to the conjectured security of the system.

- We use the RC4 stream cipher (see [6] and references in this paper) for encryption/decryption, 1st order Reed-Muller code [4 for encoding/decoding and the modified DEW system [2] to embed/extract secret information in the cover image. Even if our scheme gets cryptanalysed over time, one can choose different options in terms of available schemes to build a more robust system depending on exact hardware facility. For our specific choice we also keep in mind that the complete scheme has to be implemented on low end hardwares, e.g., mobile phones etc.

\section{Design and Implementation}

In this section we outline the design and implementation strategy of our scheme. The framework is exactly described using RC4, Reed-Muller code and the modified DEW scheme.

\section{$2.1 \quad$ RC4}

The RC4 stream cipher has been designed by Ron Rivest for RSA Data Security in 1987, and was a propriety algorithm until 1994. It uses an S-Box $S=S_{0}, \ldots, S_{255}$ of length 256, with each location of 8 bits. It is initialized as $S_{i}=i$ for $0 \leq i \leq 255$. Another array $K E Y=K E Y_{0}, \ldots, K E Y_{255}$ is used, where each location is of 8 bits. The minimum key size is 40 bits, i.e., in this case $K E Y_{0}, \ldots, K E Y_{4}$ will be filled by the key and then that is repeated number of times to fill up the entire array $K E Y$. Initially an index $j$ is set to 0 and for $(i=0 ; i<256 ; i++)\left\{j=\left(j+S_{i}+K E Y_{i}\right) \bmod 256\right.$; Swap $S_{i}$ and $\left.S_{j} ;\right\}$

The following code is used to generate a random byte: $i=(i+1) \bmod 256$; $j=\left(j+S_{i}\right) \bmod 256$;

Swap $S_{i}$ and $S_{j} ; t=\left(S_{i}+S_{j}\right) \bmod 256 ;$ keyByte $=S_{t}$;

The keyByte is XORed with the message byte to generate the cipher byte at the sender end and again the keyByte is XORed with the cipher byte to generate the message byte at the receiver end. The RC4 stream cipher has experienced a 
lot of cryptanalysis till date and it is well accepted to be used as a stream cipher for low end devices. An exact implementation of RC4 for low end sensor devices has been presented in [6] that takes only 8 machine instructions on ATMega 163L microcontroller to generate one byte of key stream after every 13 cycles. Thus it is clear that RC4 encryption methodology can be very well implemented in a mobile device since it works well for a very low end device as ATMega 163L.

\subsection{Reed-Muller Code}

The first order RM code [4] takes $(n+1)$-bit string and encodes it to $2^{n}$-bit codeword. The minimum distance between any two codewords is at least $2^{n-1}$, i.e., up to $2^{n-2}$ many errors can be corrected. The $(n+1)$-bit string $a_{0}, \ldots, a_{n}$ can be seen as an affine Boolean function $a_{0} \oplus a_{1} x_{1} \oplus \ldots \oplus a_{n} x_{n}$ on $n$-variables. The codeword corresponding to this is the $2^{n}$ length truth table corresponding to the function. During decoding one calculates the Walsh spectra given the truth table of the Boolean function which is a collection of $2^{n}$ integer values and finds out the maximum absolute value in that collection which in turn gives the affine function $a_{0} \oplus a_{1} x_{1} \oplus \ldots \oplus a_{n} x_{n}$.

For our purpose we take $n=4$, i.e., we encode a 5 -bit string to a 16 -bit codeword. This requires 16 many logical operations and 16 more memory lookup's to encode a 5-bit string to a 16-bit codeword. During decoding we need 64 many $\left(n 2^{n}\right)$ addition/subtraction operations and then finding the maximum out of 16 values. Given the low complexity of the encoding and decoding algorithm, it is clear that this strategy can be easily accommodated in a mobile device.

\subsection{Modified DEW Scheme}

Modified DEW scheme 2] embeds the message in a JPEG compressed image. It directly works with the DCT coefficients of the JPEG image. A JPEG image can be considered as a set of $8 \times 8$ DCT blocks, each block containing 64 DCT coefficients. This set is divided into different groups (known as lc-regions) and each group is further sub-divided into two sub-groups $A$ and $B$. An lc-region contains $t$ many blocks and each sub-group contains $\frac{t}{2}$ many blocks. Energy of a block is defined as the sum of $q$ many low frequency DCT coefficient values. Energy of a group or a sub-group is the sum of the energies of the constituent blocks. Energies of sub-groups $A, B$ are denoted as $E_{A}, E_{B}$. An energy difference is created among two sub-groups of a group to embed a message bit. The exact algorithm is as follows. Here the bit string to be embedded is denoted by $C_{E}=$ $c_{0}, c_{1}, \ldots, c_{l-1}$ and the $j$-th DCT coefficient of the $b$-th block is represented by $\theta_{j, b}$.

\section{Algorithm 1}

1. Randomly arrange the $8 \times 8$ DCT blocks of the JPEG image using some pseudo random generator and group them. Each group should be divided in two sub-groups such that sub-group $A$, i.e., $E_{A}$ is almost equal to the energy of sub-group B, i.e., $E_{B}$. Store this group information which we call the image key $K_{I}$. 
2. FOR $k=0$ to $l-1 D O$

(a) Select the $k$-th group consisting of $z$ blocks.

(b) Choose $\alpha_{1} \in[0,2 \alpha]$ and set $\alpha_{2}=2 \alpha-\alpha_{1}$.

(c) $I F\left(c_{k}=0\right)$ THEN

(d) $E L S E$

i. $\theta_{j, b}=\theta_{j, b} *\left(1+\alpha_{1}\right)$ for $b=1, \ldots, \frac{t}{2}-1$, and $j=1, \ldots, q$.

ii. $\theta_{j, b}=\theta_{j, b} *\left(1-\alpha_{2}\right)$ for $b=\frac{t}{2}, \ldots, t-1$, and $j=1, \ldots, q$.

i. $\theta_{j, b}=\theta_{j, b} *\left(1-\alpha_{1}\right)$ for $b=1, \ldots, \frac{t}{2}-1$, and $j=1, \ldots, q$.

ii. $\theta_{j, b}=\theta_{j, b} *\left(1+\alpha_{2}\right)$ for $b=\frac{t}{2}, \ldots, t-1$, and $j=1, \ldots, q$.

3. Arrange back the DCT blocks to their original positions and write the image.

Message extraction algorithm is blind, i.e., the extraction process does not require the original host image. In Algorithm 1 all $8 \times 8$ blocks are shuffled according to a pseudo random generator. From these shuffled blocks, the groups are chosen. The image key $K_{I}$ contains all the information about this region/subregion arrangement. Once the image key $K_{I}$ is available, one can reorganize the blocks of the stego image in the manner that was used at the time of message embedding. Now one can calculate $E_{A}, E_{B}$ for each lc-region and if $E_{A}>E_{B}$ then the recovered bit is 0 else it is 1 .

The modified DEW scheme works on JPEG images and JPEG CODEC is available in most of the processors that are used for mobile phones with camera. The LCD controllers with built in image processing circuits contain good amount of memory and processing power, e.g., S1D13710/712 (see EPSON ED Journal, Vol 30, 2002) has embedded memory of 224/320 KBytes, and the CPU is of 16bit with $32.768 \mathrm{kHz}$ clock. Thus it looks feasible to implement modified DEW on a mobile device efficiently.

\subsection{Experimental Results}

The exact implementation of our complete scheme on low end devices is at its early stage. Sun Microsystems Java2 micro edition (J2ME) with Mobile Information Device Profile (MIDP) is one of the most used platforms for developing hardware and OS independent applications on mobile devices (see http://developers.sun.com/techtopics/mobility/midp/). The Symbian Application Development environment (see http://www.symbian.com) is also a popular platform. We are currently studying the feasibility of the implementation on such platforms.

The experimental result presented below is based on a standard $\mathrm{C}$ code implementation of the complete scheme on LINUX operating system in a PC. This has been done to study the the complete implementation and available from the first author on email request for public evaluation. In our experiment a message of length 10 characters (here the message is "attackOn8P") is embedded in the Lena image of size $512 \times 512$. The message is of $10 \cdot 8=80 \mathrm{bits}$ and encrypted using RC4 algorithm with a 40-bit key. The encrypted message is encoded using $\mathrm{RM}$ error-correcting code and generates $\frac{80}{5} \cdot 16=256$ bits. These 256 bits are embedded in the Lena image. Since the image is of size $512 \times 512$, we have total 
$\frac{512 \times 512}{8 \times 8}=4096$ many DCT blocks. The binary string to be embedded is 256 bits, so there are $\frac{4096}{256}=16$ many DCT blocks in each lc-region, i.e., each sub-group contains 8 many DCT blocks. The experimental parameters for Algorithm 1 are as follows: $\alpha=.05, \alpha_{1}=0, \alpha_{2}=2 \alpha=0.1, q=5, t=16, l=256$.

The image containing the secret information is of very high visual quality and the PSNR of the stego image is $41.6 \mathrm{~dB}$ with respect to the original image. The method can withstand image processing attacks, compression and filtering operations. As example, if the stego (or watermarked) image is re-compressed using a very low 10\% JPEG quality, then also we can recover the message correctly. The comparison of our scheme with other schemes will be available in the full version of this paper.

\section{Conclusion}

Here we have presented a basic framework for information hiding on mobile devices with camera. For the basic assumption of steganography, the sender and receiver will share the secret private keys $K, K_{I}$ at an earlier instance than when the exact transmission of the image takes place, i.e., there is no communication other than the image itself that contains the secret information. Thus, the cover image $I$ and the corresponding image key $K_{I}$ need to be fixed at an earlier instance while deciding the key $K$. This presents a restriction on online choice of the cover image. On the other hand, for the purpose of watermarking this is not at all a problem as the owner will store all the key information with him/her to prove the copyright and only the watermarked image will be communicated. Thus for watermarking application, choice of image could be online without any restriction.

\section{References}

1. R. J. Anderson and F. A. P. Petitcolas. On The Limits of Steganography. IEEE Journal of Selected Areas in Communications. Special Issue on Copyright and Privacy Protection, 16(4):474-481, May 1998.

2. T. K. Das, S. Maitra and J. Mitra. Cryptanalysis of Optimal Differential Energy Watermarking (DEW) and a Modified Robust Scheme. IEEE Transactions on Signal Processing, 53(2):768-775, February 2005.

3. S. Katzenbeisser, F. A. P. Petitcolas (edited). Information Hiding Techniques for Steganography and Digital Watermarking. Artech House, USA, 2000.

4. F. J. MacWillams and N. J. A. Sloane. The Theory of Error Correcting Codes. North Holland, 1977.

5. L. M. Marvel, C. G. Boncelet, Jr and C. T. Retter. Reliable Blind Information Hiding for Images. In Information Hiding 1998, LNCS 1525, Pages 48-61, 1998.

6. A. Seshadri, A. Perrig, L. v. Doorn, P. Khosla. SWATT: SoftWare-based ATTestation for Embedded Devices. IEEE Symposium for Security and Privacy, 2004. 